\title{
Primary pneumomediastinum in a young woman with near fatal asthma. Diagnosis by ultrasound: a
} case report

\section{Abstract}

We present the case of a female patient presenting with severe asthma crisis, with evolution to severe respiratory failure due to primary pneumomediastinum. The early diagnosis of this pathology was made through the use of ultrasound, where we discard pneumotorax as the etiology of decompensation. The diagnosis was subsequently corroborated by Thorax Computed Tomography (CT), as gold standard.

\author{
Volume 5 Issue 3 - 2018 \\ Vásquez-Tirado Gustavo, ${ }^{1,2,3,4}$ Evangelista- \\ Montoya Félix ${ }^{1,3,4}$ \\ 'Medicina Interna, Peru \\ 2 Medicina Intensiva, Peru \\ ${ }^{3}$ Servicio de Unidad de Cuidados Intensivos, Peru \\ ${ }^{4}$ Hospital Regional Docente de Trujillo, Peru
}

\begin{abstract}
Correspondence: Vásquez-Tirado Gustavo, Medicina Interna, Medicina Intensiva, Servicio de Unidad de Cuidados Intensivos, Hospital Regional Docente de Trujillo, Unidad de Posgrado, Universidad Privada Antenor Orrego, Trujillo, Peru, Tel +51947474019, Email gustavovat@msn.com
\end{abstract}

Received: March 23, 2018 | Published: May 21, 2018

\section{Introduction}

The current use of ultrasound like skill of diagnosis on the first assesment to critically ill patient plays a fundamental role for start and to guide a correct therapy. Diverse approach protocols, like the BLUE protocol in acute respiratory failure, have been established which aim through algorithms to approach the diagnosis in a patient with that illness.

It easy access, speed, little o no radiation and, low cost make it ultrasound a fundamental resource in critical areas. ${ }^{1}$ The primary pneumomediastinum is a very rare entity; some series of cases report an incidence of 1: 7000 to 1: 45,000 admissions to emergency, predominantly in females; Although usually benign and self-limited, is often due to a patient's life-threatening condition. ${ }^{2}$

We present the case of a young female patient who was admitted due to a severe asthmatic crisis that evolves unfavorably, in which the diagnosis of pneumomediastinum was performed by ultrasonography, ruling out the presence of pneumothorax.

\section{Clinical case}

An 18-year-old woman with a diagnosis of bronchial asthma from 4 years old, irregular treatment, five years ago was admitted to the Intensive Care Unit (ICU) due to acute respiratory failure. He comes to the Emergency Department for dyspnea and wheezing of 12 hours of evolution that progresses despite the use of inhaled salbutamol.

During crisis treatment with salbutamol in nebulization she perceived severe sudden pain in the left sternoclavicular region and sensation of oppression in the neck, dysphonia which made breathing even more difficult. There is evidence of swelling and crepitation in the neck, compatible with subcutaneous emphysema. With a SonoAce X8 (Samsung Medison) machine, with convex and phased array probes, an ultrasound evaluation was performed according to the BLUE Protocol to guide the etiology of decompensation in the context of near-fatal asthma, finding that there is adequate pleural sliding in both hemithorax, which discards the presence of bilateral pneumothorax (Figure 1); It is worth noting the impossibility of visualizing the heart in different windows by the interposition of like B-lines artefacts that start from hyperechogenic points in the projection of the cardiac border, suggestive of air (Figure 2), which raises the diagnosis of Pneumomediastinum. When evaluating the anterior cervical region (with linear probe), like B-lines artefacts are visualized, which prevent the partial visualization of neck structures compatible with subcutaneous emphysema (Figure 3), thereby reinforcing the diagnosis. A chest X-ray is performed (Figure 4), where there is a thin radiolucency on the left cardiac border, a suspected pneumomediastinum. Four hours later, thoracic CT was performed confirming pneumomediastinum (Figure 5).

Patient subsequently presented a new episode of pain with a higher oxygen requirement, which led to invasive mechanical ventilation and ICU admission. Four days later, when the ultrasound is performed again in the patient, a decrease in subcutaneous emphysema is verified, a partial view of cardiac cavities and the Air Gap Sign (Figure 6) are visualized. After 10 days, the patient is extubated and goes to NonInvasive Ventilation for 8 hours, according to our protocols, after which it presents good tolerance and indicates ICU discharge.

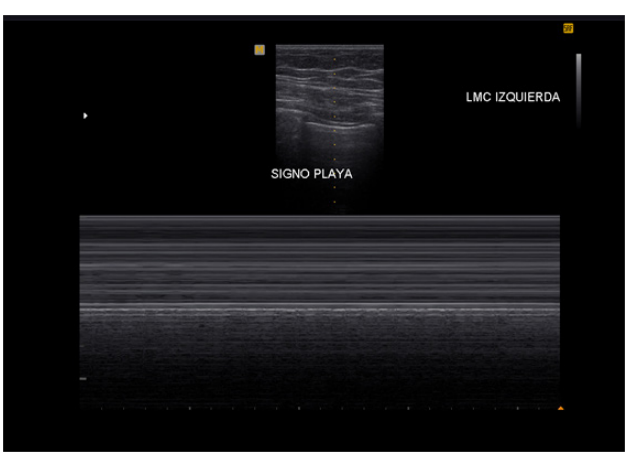

Figure I Pulmonary ultrasound, evidence consistent with lung sliding. In $M$ mode the normal sea-shore pattern, which discards pneumothorax as a diagnosis. 


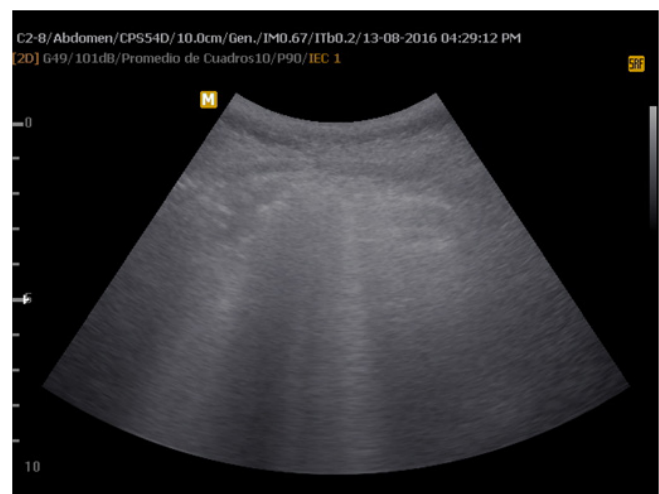

Figure 2 Echocardiography, apical 4 chambers view. It is not possible to delineate the heart structure, in its place, images similar to $B$ pleural lines which radiate from the parietal pericardium

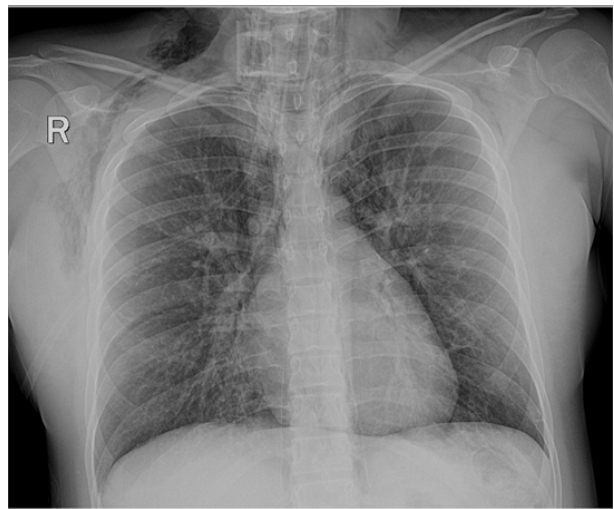

Figure 4 Anteroposterior thoracic radiograph. Radiolucency was observed around the heart, consistent with suspected pneumopericardium.

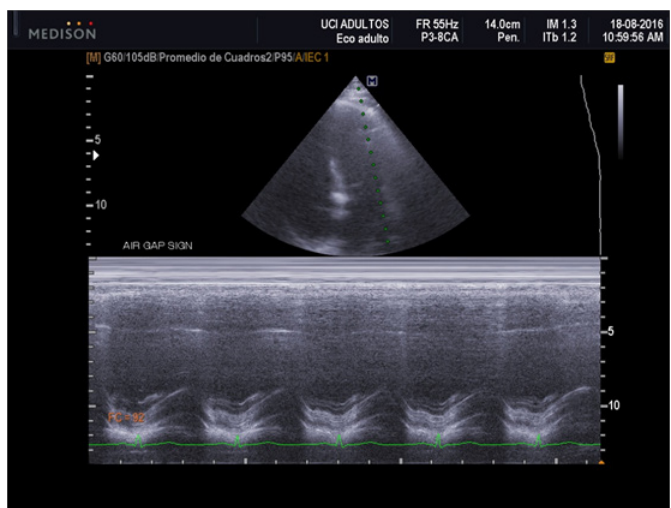

Figure 6 Echocardiography, parasternal long axis view. In mode M, at the level of the mitral valve, the air gap sign is observed, indicating pneumomediastinum.

\section{Discussion}

Spontaneous pneumomediastinum or Hamman's syndrome is defined by Yellin as the accumulation of air at the level of the mediastinum without any apparent predisposing factor, such as having mediated some chest trauma, tracheoesophageal procedures or mechanical ventilation, also without relation to cardiac catheterization or thoracic surgery. As in the case of the patient described, she presents abruptly with severe chest pain, aggravation of dyspnea already present and cervical subcutaneous emphysema. ${ }^{3}$

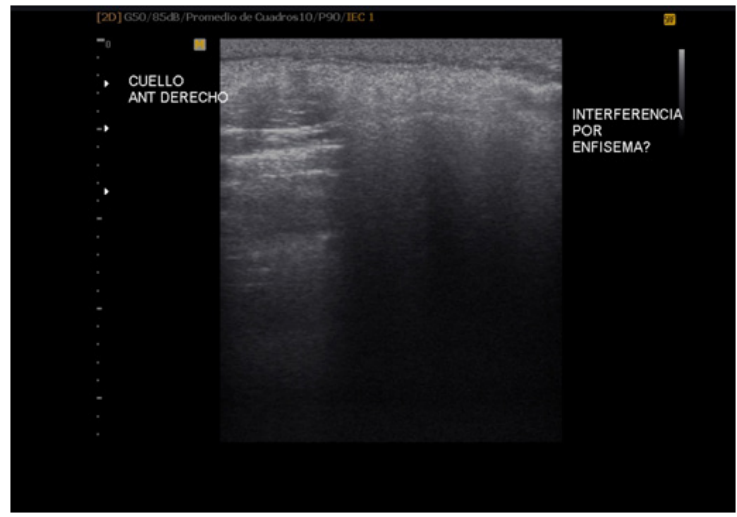

Figure 3 Soft tissue ultrasound of the anterior neck. Images are consistent with cervical emphysema.

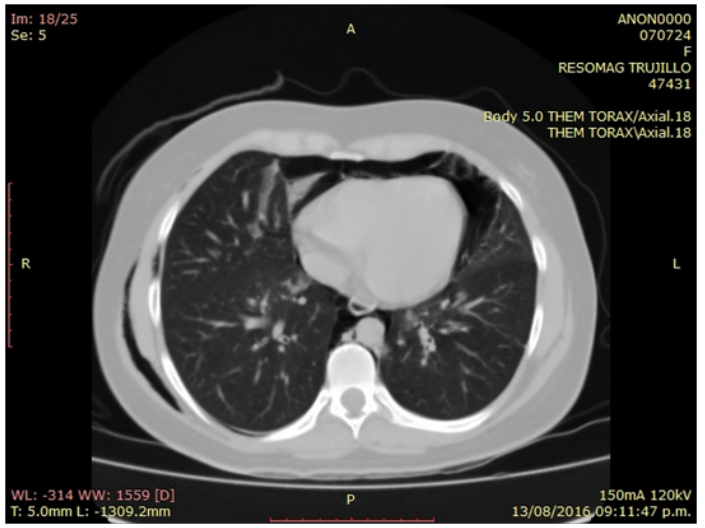

Figure 5 Thoracic tomography, indicating pneumopericardium and pneumomediastinum.

In 1939, Louis Hamman describes this pathology, from which becomes the sign that bears his name, which is the crepitation of synchronous auscultation with the heart beat in systole at the level of the anterior region of the thorax, specifically in the left parasternal border (Hamman's sign, pathognomonic of this pathology, present only in 10 to $20 \%$ of the cases). ${ }^{3,4}$

The pathophysiological explanation of the primary spontaneous pneumomediastinum is done in 1944, by Macklin C and Macklin M, with the so-called Macklin effect, where they postulate that the increase of the intrathoracic pressure is translated, in turn, in increase of intraalveolar pressure, resulting in rupture of the terminal alveoli with the consequent release of air to the pulmonary interstitium, migrating through the sheath of the pulmonary vessels to the mediastinum. And so the air can extend into the subcutaneous tissue of the chest wall and neck forming characteristic subcutaneous emphysema. ${ }^{4}$

The precipitating factors associated with increased intraalveolar pressure are related to patients in asthmatic crisis, such as in our patient, and others such as vomiting, Valsalva maneuver, cough, defecation, intense physical exercise and associated with difficult delivery. On the other hand the predisposing factors are asthma, interstitial diseases, inhalation of some illicit drugs like cocaine, marijuana or other irritating substances. ${ }^{2,4}$

Although the papers that report series of cases begin the diagnostic study with the simple chest $\mathrm{x}$-ray, the gold standard of diagnosis continues to feel chest $\mathrm{CT}^{2-4}$ In our patient, the initial approach was 
performed with thoracic ultrasound. Tension pneumothorax was ruled out as a cause of decompensation and emphysema; observing the ultrasound findings compatible with pneumomediastinum, finally corroborated by chest CT. Within the first ultrasound description of the pneumomediastinum, Reid et al. ${ }^{5}$ In 1983 they make the echographic description of "air gap sign" as a diagnosis of pneumomediastinum. It consists of evidence of a cyclical loss of the echocardiographic image that begins in systole and continues until early diastole.

Some extend to definition by saying that such loss of signal would also be evidenced in $\mathrm{M}$ and bidimensional modes. ${ }^{5,6}$ The important features are described: a band of echoes within the cardiac chamber beginning at the anterior cardiac border due to air accumulation; total dropout of echoes posteriorly; and, the cyclic appearance of this air gap sign..$^{5,7}$

In our patient, at the time of admission, an echocardiographic window in the apical region with 4-chamber view reveals a structure that beats but was impossible recognize the heart structure, only like-B pleural lines type artefacts (Figure 2), which It would correspond to a pneumomediastinum covering the entire anterior part of the heart. Later in the evolution of the patient, with the clinical improvement, was possible to see the air gap sign (Figure 6), which is corrobored in the thorax $\mathrm{CT}$, where we saw pneumomediastinum and pneumopericardium.

When we speak exclusively of pneumopericardium we must find mainly two characteristics in the ultrasound that are the presence of comet tails that starts from the parietal pericardium and, the cyclic disappearance of the heart in some points during the systole. ${ }^{8-10}$ As we can see, both the pneumomediastinum and the pneumopericardium share some echographic similarities, except that they show inclusion in the pericardial space as such air bubbles. In the case of hydropneumopericardium the presence of bright bubbles in the pericardial space that have the same cyclic disappearance characteristics are described. ${ }^{10}$ As in our patient, in those with acute respiratory failure with a high suspicion of pneumomediastinum, ultrasound has high diagnostic sensitivity, which would allow us an early therapeutic approach.

\section{Conclusion}

The presence in echocardiography of cardiac images with cyclic disappearance in systole, both in M-mode and B-mode (air gap sign) in addition to the presence of comet tails like artifacts originating from the parietal pericardium, are highly suspected of pneumomediastinum.

\section{Acknowledgements}

None.

\section{Conflict of interest}

The author declares that there is no conflict of interest.

\section{References}

1. Lichtenstein DA, Meziére GA. Relevance of lung ultrasound in the diagnosis of acute repiratory failure: The BLUE protocol. Chest. 2008;134(1):117125 .

2. Álvarez C, Jadue A, Rojas F, et al. Neumomediastino espontáneo (síndrome de Hamman): Una enfermedad benigna mal diagnosticada. Rev Méd Chile. 2009;137:1045-1050.

3. Meireles J, Neves S, Castro A, et al. Spontaneous pneumomediastinum revisited. Respiratory Medicine CME. 2011;4(4):181-183.

4. Newcomb A, Clarke P. Spontaneous Pneumomediastinum : A Benign Curiosity or a Significant Problem? Chest. 2005;5(128):3298-3302.

5. Reid C, Chandraratna A, Kawanishi D, et al. Echocardiographic detections of pneumomediastinum and Pneumopericardium: The Air Gap Sign. $J$ Am Coll Cardiol. 1983;1(3):916-921.

6. Kerut E, Hannawalt C, Everson C, et al. The Air Gap Sign. Echocardiography 2014:1-2.

7. Uluçam M. An extremely rare combination: pneumopericardium, pneumoperitoneum and subcutanous emphysema - a case report. Cardiol Ther. 2013;2(1):013-110.

8. Testa A, Candelli M, Pignataro G, et al. Sonographic detection of spontaneous pneumomediastinum. J Ultrasound Med. 2008;27:1507-1509.

9. Zachariah S, Gharahbaghian L, Perera P, et al. Spontaneous pneumomediastinum on bedside ultrasound: case report and review of the literatura. West J Emerg Med. 2015;2(16):321-324.

10. Bobbia X, Claret P, Muller L, et al. Pneumopericardium diagnosis by pointof-care Ultrasonography. J Clin Ultrasound. 2012;41(4):1-3. 